\title{
Comportamiento de los procesos sépticos cervicofaciales en pacientes hospitalizados en el servicio de cirugía maxilofacial
}

\section{Behaviour of septic processes of the head and neck in patients hospitalized in the department of maxillofacial surgery}

\author{
A.C. Valdez Borroto ${ }^{1}$, L.D. Medina Vega' ${ }^{1}$ W. Portal Fernández' ${ }^{1}$ J. Martín Pino², \\ P.L. Gutiérrez Martínez ${ }^{3}$
}

Resumen: Se realizó un estudio descriptivo prospectivo de los 243 pacientes ingresados con procesos sépticos cervicofaciales en el Servicio de Cirugía Maxilofacial del Hospital Provincial Universitario "Arnaldo Milián Castro" de Santa Clara, Villa Clara, en el período de enero de 1999 a diciembre de 2000, con el propósito de mostrar el comportamiento clínico, epidemiológico y terapéutico de los mismos. El 20,3\% de los ingresados se identificó como proceso séptico, de los cuales el 53,1\% resultó no odontógeno. El grupo de edades más afectado fue el de 20 a 39 años, así como el sexo masculino. Las localizaciones anatómicas mayormente afectadas fueron la geniana y la submandibular. La instauración de un tratamiento antimicrobiano y complementario adecuado favoreció en la mayoría de los pacientes una estadía hospitalaria de 1 a 7 días. En la casi totalidad de los enfermos, la evolución resultó favorable dada la no existencia de complicaciones.

Palabras clave: Infeccion focal/epidemiología; Infeccion focal dental; Infeccion focal/Complicaciones; Antibioticos/uso terapéutico.

Recibido: 12.04 .04

Aceptado: 06.10.06
1 Especialista de Primer Grado en Cirugía Maxilofacial. Hospital Universitario Arnaldo Milián Castro. Villa Clara. Cuba.

2 Doctora en Estomatología. Clínica Estomatológica: Celia Sánchez Manduley. Santa Clara. Villa Clara. Cuba.

3 Especialista de Primer Grado en Ortopedia y Traumatología. Hospital Universitario Arnaldo Milián Castro. Villa Clara. Cuba.

\section{Correspondencia:}

Luis Daniel Medina Vega

Prolongación de Martha Abreu, 100 entre B y C

Reparto Virginia. Santa Clara. Villa Clara, Cuba

E-mail: Luisdaniel@cubasi.cu
Abstract: A descriptive and prospective study was carried out on 243 patients admitted with the diagnosis of septic cervicofacial processes to the University Hospital "Arnaldo Milián Castro" during 1999 to 2000, in order to establish the epidemiological, clinical, and therapeutic features of this entity in these patients. A septic process was identified in $20.3 \%$ of the total number of patients admitted; $53.1 \%$ of these were of a non-odontogenic origin. There was a prevalence among males and among the 21-30 age group. The anatomic regions most affected were the genian and submandibular areas. Most patients were hospitalized between one and seven days. A minimal complication rate was reported.

Key words: Focal infection/epidemiology; Focal infection dental; Focal infection/complications; Antibiotics/therapeutic use. 


\section{Introducción}

Las enfermedades infecciosas han influido de manera intensa en la evolución de la historia humana. En la época medieval se recuerda la pandemia provocada por la peste negra que logró cambiar las estructuras sociales de la Europa de entonces. Incluso, muchas campañas militares vieron alterados sus resultados por brotes de enfermedades como la disentería y el tifus. ${ }^{1}$

Las enfermedades infecciosas son en estos momentos la principal causa de mortalidad y morbilidad en el mundo en desarrollo. ${ }^{2}$ Su reconocimiento, la conformación de su etiología y las actitudes terapéuticas han sido motivos de ardua investigación para dominarlas.

Las infecciones cervicofaciales constituyen una enfermedad de gran importancia, tanto por su elevada frecuencia, como por su potencial gravedad. Ocupan aproximadamente entre 20 y $25 \%$ de los pacientes atendidos en los servicios de Cirugía Maxilofacial. ${ }^{3}$

Se definen dos vertientes etiológicas en la región, individualizadas clínica y terapéuticamente: los procesos de origen odontógenos y los no odontógenos.

Las infecciones no odontogénicas excluyen el origen dentario y son secundarias a infecciones glandulares, sinusales, posquirúrgicas, postraumáticas, cutáneas y mucosas, sobre infecciones de neoplasmas, quistes branquiales y dermoides entre otros. ${ }^{4,5}$

La infección odontógena es aquella cuyo origen es la estructura que forma el diente y el periodonto. Es el tipo de infección más común en la región cervicofacial.6,7

Llama la atención el elevado número de pacientes que acude a nuestro cuerpo de guardia con esta enfermedad y un gran porcentaje de ellos requiere hospitalización. Es de destacar que un tratamiento inadecuado en el área de salud ha condicionado la perpetuación y exacerbación de la enfermedad en muchos de los aquejados.

Lo antes expresado ha motivado nuestro interés en estudiar clínica, epidemiología y terapéuticamente la enfermedad cervicofacial con el propósito de dar a conocer su comportamiento en nuestro medio.

\section{Material y método}

Se realizó un estudio descriptivo prospectivo de los 243 pacientes con procesos sépticos cervicofaciales que ingresaron en el servicio de Cirugía Maxilofacial del Hospital Provincial Universitario "Arnaldo Milián Castro" de Santa Clara, Villa Clara, Cuba, en el período de enero de 1999 a diciembre de 2000 y cuya selección estuvo dada por la totalidad (universo) de dichos pacientes.

Para la recogida de la información se confeccionó un formulario (ver anexos) con las variables de interés, las cuales se obtuvieron por el método de encuesta, mediante la observación clínica del paciente y la revisión de las clínicas individuales confeccionadas al ingreso y archivadas en el Departamento de Estadísticas de la institución hospitalaria. Dichas variables fueron: tipo de proceso séptico, sexo, edad, localizaciones anatómicas, antimicrobianos utilizados, estancia y complicaciones.

\section{Introduction}

Infectious diseases have influenced the evolution of human history intensely. In the medieval era, the pandemic caused by the black plague changed the social structure of Europe at that time. Even the results of many military campaigns were altered as a result of the outbreak of diseases such as dysentery and typhus.'

Infectious diseases are at this moment the principal cause of mortality and morbidity in the developed world. ${ }^{2}$ Recognizing these, the conformation of their etiology and therapeutic attitudes have motivated the arduous investigation in order to control them.

Head and neck infections represent a disease of great importance, as much for their high incidence as for how potentially serious they can be. They make up approximately 20 to $25 \%$ of the patients attended by the departments of Maxillofacial surgery. ${ }^{3}$

Two etiological aspects in the region can been defined, and individualized clinically and therapeutically: odontogenic and non-odontogenic processes.

Non-odontogenic infections exclude a dental origin and they are secondary to glandular, sinusal, postsurgical, posttraumatic, cutaneous and mucosal infections, extra infections from neoplasms, branchial and dermoid cysts amongst others. ${ }^{4-5}$

Odontogenic infection originates in the structure that forms the tooth and periodontium. It is the most common type of infection in the head and neck region. ${ }^{6-7}$

Of note is the high number of patients that attend our on-duty service with this disease, and many require hospitalization. It should be pointed out that inadequate treatment by a health center has led to the perpetuation and exacerbation of the disease in many sufferers.

All this has lead to our interest in studying head and neck disease clinically, epidemiologically and therapeutically with the aim of reporting on its behavior in our area.

\section{Material and method}

A prospective descriptive study was carried out of 243 patients with septic processes of the head and neck who were admitted into the department of Maxillofacial Surgery of the "Hospital Provincial Universitario Arnaldo Milián Castro" in Santa Clara, Villa Clara, Cuba, between January 1999 and December 2000. The selection was made up of entirely of these patients.

A form was drawn up for data collection (see annex) with the variables of interest. These had been obtained by carrying out a survey based on the clinical observation of the patient and on reviewing the medical records individually. These had been drawn up on admittance and filed in the Statistics Department of the hospital. The variables were: type of septic process, sex, age, anatomic location, type of antimicrobial agent used, stay and complications. 
Los datos se computarizaron y procesaron mediante el sistema SPSS8.0 para Windows donde se estructuró una base de datos. Dichos datos se resumieron en tablas estadísticas. Se determinaron frecuencias absolutas (número de pacientes) y relativas (porcentajes) en las distribuciones de frecuencia conformadas.

Para el análisis e interpretación de los resultados se utilizó el análisis porcentual, la media aritmética y la desviación estándar y se aplicaron pruebas estadísticas inferenciales. Para determinar si existían relaciones significativas entre las variables, se utilizó la prueba de Chi Cuadrado y se mostró como resultado del mismo el valor del estadígrafo $x^{2} y$ su significación asociada $p$.

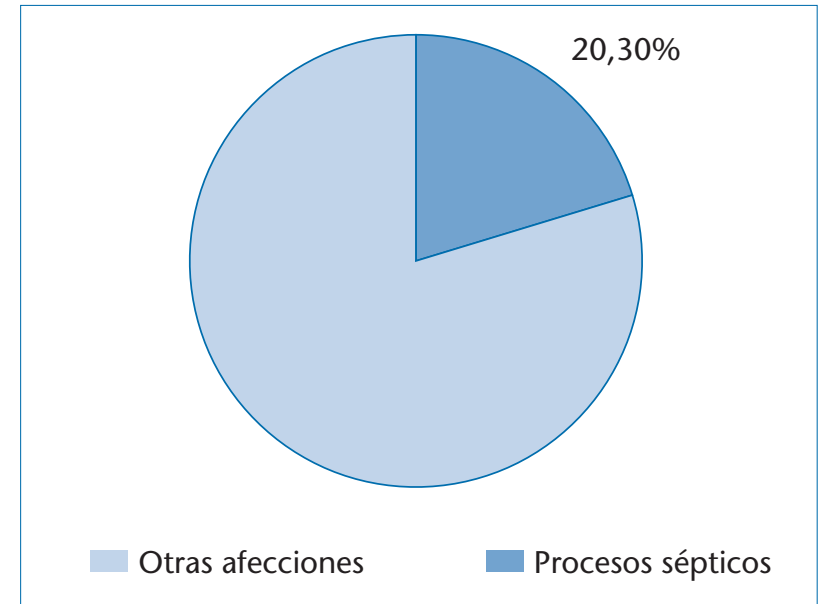

Figura 1. Incidencia de procesos sépticos. Hospital provincial universitario"Arnaldo Milián Castro" (Fuente: Historias clínicas individuales).

Figure 1. Incidence of septic processes. "Hospital Provincial Universitario Arnaldo Milián Castro" (Source: Individual medical records).
The data was fed into a computer and processed with the Windows SPSS8.0 system and a database was set up. The data were summarized in statistics tables. Absolute frequencies were determined (number of patients) and relative (percentages) in the distribution of adjusted frequencies.

Percentage analysis, arithmetic average and standard deviation were used for the analysis and interpretation of the results, and inferential statistics were applied. In order to determine if a significant link between the variables existed, the Chi-square test was used and the result given was that of the same value of the statistic $x^{2}$ and its associated significance $p$.

\section{Results}

The incidence of septic processes is reflected in figue 1. Patients with infectious diseases make up $20.3 \%$ of the total number of inpatients, which is a fifth of the total.

On analyzing the septic process with regard to sex and etiology (Table 1), a predominance of non-odontogenic infections can be detected (53.1\%), but in general they keep relative equity with odontogenic processes. More than half the processes corresponded to the male sex (54.3\%) and most of the patients with non-odontogenic septic processes were

Tabla 1. Distribución de los procesos sépticos según sexo

\begin{tabular}{|c|c|c|c|c|c|c|}
\hline & \multicolumn{2}{|c|}{ Odontógenos } & \multicolumn{2}{|c|}{ No odontógenos } & \multicolumn{2}{|c|}{ Total } \\
\hline & $\mathbf{N}^{\circ}$ & $\%$ & $\mathbf{N}^{\circ}$ & $\%$ & $\mathbf{N}^{\mathbf{O}}$ & $\%$ \\
\hline Masculino & 51 & 44,7 & 81 & 62,8 & 132 & 54,3 \\
\hline Total & 114 & 46,9 & 129 & 53,1 & 243 & 100,0 \\
\hline
\end{tabular}

Table 1. Distribution of septic processes according to sex

\begin{tabular}{lcc} 
& \multicolumn{2}{c}{ Odontogenic } \\
& No & \% \\
Masculine & 51 & 44.7 \\
Feminine & 63 & 55.3 \\
Total & 114 & 46.9
\end{tabular}

No Odontogenic
$\mathbf{N}^{\circ}$

Total

$\begin{array}{cc}\text { No } & \text { \% } \\ 132 & 54.3 \\ 111 & 45.7 \\ 243 & 100.0\end{array}$

$p<0.005 ; x^{2}=7.94957 ; D F=1$ (Source: Individual medical records). 
Tabla 2. Distribución de los procesos sépticos según la edad

\begin{tabular}{|c|c|c|c|c|c|c|}
\hline \multirow[b]{2}{*}{ Edad (en años) } & \multicolumn{2}{|c|}{ Odontógeno } & \multicolumn{2}{|c|}{ No odontógeno } & \multicolumn{2}{|c|}{ Total } \\
\hline & $\mathbf{N}^{\circ}$ & $\%$ & $\mathbf{N}^{\circ}$ & $\%$ & $\mathbf{N}^{\circ}$ & $\%$ \\
\hline Menos de 20 años & 21 & 18,4 & 12 & 9,3 & 33 & 13,6 \\
\hline $40-59$ & 20 & 17,5 & 34 & 26,4 & 54 & 22,2 \\
\hline 60 ó más & 3 & 2,6 & 17 & 13,2 & 20 & 8,2 \\
\hline Total & 114 & 46,9 & 129 & 53,1 & 243 & 100,0 \\
\hline
\end{tabular}

Table 2. Distribution of septic processes according to age

\begin{tabular}{lcc} 
& \multicolumn{2}{c}{ Odontogenic } \\
Age (years) & $\mathbf{N}^{\mathbf{0}}$ & $\%$ \\
Under 20 & 21 & 18.4 \\
$20-39$ & 70 & 61.4 \\
$40-59$ & 20 & 17.5 \\
60 or over & 3 & 2.6 \\
total & 114 & 46.9 \\
$p<0.005 ; x^{2}=15.13356 ; D F=3$ & (Source: Individual medical records).
\end{tabular}

\begin{tabular}{cccc}
$\begin{array}{l}\text { No Odontogenic } \\
\text { No }\end{array}$ & \multicolumn{2}{c}{ Total } \\
$\mathbf{N}^{\mathbf{2}}$ & 9.3 & $\mathbf{N}^{\circ}$ & $\%$ \\
12 & 51.2 & 33 & 13.6 \\
66 & 26.4 & 136 & 56.0 \\
34 & 13.2 & 54 & 22.2 \\
17 & 53.1 & 20 & 8.2 \\
129 & & 243 & 100.0
\end{tabular}

$p<0.005 ; x^{2}=15.13356 ; D F=3$ (Source: Individual medical records).

ticos, lo que indica que el sexo influye en el tipo de proceso $\left(x^{2}=7,9496 ; G L=1\right)$.

La tabla 2 se refiere a la distribución de los procesos sépticos según la edad. En nuestro estudio se tuvo en consideración un amplio rango de edades (15-84), pero la mayoría de los pacientes correspondieron al grupo de 20-39 años (56\%) que también predominó para cada tipo de proceso $(61,4 \%$ para los odontógenos y $51,2 \%$ para los no odontógenos).

La comparación estadística entre la edad y el tipo de proceso séptico resulta muy altamente significativa $(p=0,00171)$, muestra que la edad influye en la aparición de la sepsis cervicofacial $\left(x^{2}=15,13356 ; G L=3\right)$.

Las localizaciones anatómicas más frecuentes en los procesos sépticos se enumeran en la tabla 3; como se aprecia, hubo un predominio de las regiones genianas y submandibular con $45,7 \%$ y $43,2 \%$ respectivamente; asimismo, los procesos odontógenos fueron superiores en la región geniana $(48,8 \%)$ aunque en esta última las diferencias no son significativas. Hubo una relación muy altamente significativa $(p<0,001)$ entre las localizaciones anatómicas submandibular, maseterina, mentoniana, de cuerpo mandibular y parótida con los procesos sépticos $\left(x^{2}=28.967 ; x^{2}=21.717 ; x^{2}\right.$ =22.886; $x^{2}=11.155 ; G L=1$ ).

En la tabla 4 se exponen las complicaciones presentadas por los pacientes según tipo de proceso. La mayoría de ellos no tuvo complicaciones $(95,9 \%)$, lo que habla a favor de la combinación antimicrobiano-drenaje. Llama la atención que en los procesos odontógenos las complicaciones fueron consecuencia de la extensión local de la enfermedad (por continuidad); en los procesos no odontógenos la propagación a distancia fue la responsable de todas las complicaciones.

La estrategia hospitalaria evidentemente conduce al profesional a conocer la efectividad del tratamiento instaurado. La males (62.8\%). The relationship between sex and etiology in septic processes is highly significant $(p=0.00481)$, which indicates that sex influences the type of process $(x 2=7.9496$; $D F=1)$.

Table 2 shows the distribution of septic processes according to age. In our study the wide age range was taken into account (15-84), but most patients corresponded to the 2039 age group (56\%), and they also predominated each type of process (61.4\% for odontogenic and $51.2 \%$ for non-odontogenic). The statistical comparison between age and type of septic process is highly significant ( $p=0.00171)$, and it shows that age influences the appearance of head and neck sepsis $\left(x^{2}=15.13356 ; D F=3\right)$.

The most common anatomic locations in septic processes are set out in table 3. As can be appreciated, there was a predominance in the genian and submandibular regions of $45.7 \%$ and $43.2 \%$ respectively. Thus, the odontogenic processes were greater in the genian region. (48.8\%) although here the differences were not significant. There was a highly significant relationship $(p<0,001)$ between the anatomic locations that were submandibular, masseteric, genian, of the mandibular body and the parotid region with septic processes $\left(x^{2}=28.967 ; x 2=21.717 ; x^{2}=22.886 ; x^{2}\right.$ $=11.155 ;$ DF =1).

Table 4 shows the complications presented by patients according to the type of process. Most of these did not have complications (95.9\%), which speaks in favor of the antimicrobial-drainage combination. Of note in the odontogenic processes were the complications as a consequence of the local extension of the disease (because of continuity). In the non-odontogenic processes, distant propagation was responsible for all the complications. 
Tabla 3. Localizaciones anatómicas más frecuentes en los procesos sépticos

\begin{tabular}{|c|c|c|c|c|c|c|}
\hline \multirow{2}{*}{$\begin{array}{l}\text { Localizaciones } \\
\text { anatómicas }\end{array}$} & \multicolumn{2}{|c|}{ Odontógeno } & \multicolumn{2}{|c|}{ No odontógeno } & \multicolumn{2}{|c|}{ Total } \\
\hline & $\mathbf{N}^{\mathbf{0}}$ & $\%$ & $\mathbf{N}^{\circ}$ & $\%$ & $\mathbf{N}^{\circ}$ & $\%$ \\
\hline Geniana & 48 & 42,1 & 63 & 48,8 & 111 & 45,7 \\
\hline Submandibular** & 70 & 61,4 & 35 & 27,1 & 105 & 43,2 \\
\hline Periorbitaria & 21 & 18,4 & 34 & 26,4 & 55 & 22,6 \\
\hline Nasolabial & 17 & 14,9 & 29 & 22,5 & 46 & 18,9 \\
\hline Submental* & 25 & 21,9 & 16 & 12,4 & 41 & 16,9 \\
\hline Maseterina*** & 26 & 22,8 & 4 & 3,1 & 30 & 12,3 \\
\hline Mentoniana*** & 4 & 1,5 & 19 & 14,7 & 23 & 9,5 \\
\hline Cuerpo mandibular*** & 21 & 18,4 & 1 & 0,8 & 22 & 9,1 \\
\hline Cavidad oral & 13 & 11,4 & 8 & 6,2 & 21 & 8,7 \\
\hline Zigomática & 9 & 7,9 & 10 & 7,8 & 19 & 7,8 \\
\hline Cervical lateral & 7 & 6,1 & 7 & 5,4 & 14 & 5,7 \\
\hline Frontotemporal* & 2 & 1,8 & 11 & 8,5 & 13 & 5,3 \\
\hline Parotídea*** & - & - & 12 & 9,3 & 12 & 4,9 \\
\hline Auricular** & - & - & 8 & 6,2 & 8 & 3,2 \\
\hline
\end{tabular}

Table 3. Most common anatomic location of septic process

\begin{tabular}{|c|c|c|c|c|c|c|}
\hline \multirow[b]{2}{*}{ Anatomic location } & \multicolumn{2}{|c|}{ Odontogenic } & \multicolumn{2}{|c|}{ No Odontogenic } & \multicolumn{2}{|c|}{ Total } \\
\hline & $\mathbf{N}^{\circ}$ & $\%$ & $\mathbf{N}^{\circ}$ & $\%$ & $\mathbf{N}^{\mathbf{0}}$ & $\%$ \\
\hline Genian & 48 & 42.1 & 63 & 48.8 & 111 & 45.7 \\
\hline Submandibular** & 70 & 61.4 & 35 & 27.1 & 105 & 43.2 \\
\hline Periorbital & 21 & 18.4 & 34 & 26.4 & 55 & 22.6 \\
\hline Lip/nose & 17 & 14.9 & 29 & 22.5 & 46 & 18.9 \\
\hline Submental* & 25 & 21.9 & 16 & 12.4 & 41 & 16.9 \\
\hline Masseter*** & 26 & 22.8 & 4 & 3.1 & 30 & 12.3 \\
\hline Chin*** & 4 & 1.5 & 19 & 14.7 & 23 & 9.5 \\
\hline Mandibular body*** & 21 & 18.4 & 1 & 0.8 & 22 & 9.1 \\
\hline Oral cavity & 13 & 11.4 & 8 & 6.2 & 21 & 8.7 \\
\hline Zygomatic & 9 & 7.9 & 10 & 7.8 & 19 & 7.8 \\
\hline Lateral cervical & 7 & 6.1 & 7 & 5.4 & 14 & 5.7 \\
\hline Frontotemporal* & 2 & 1.8 & 11 & 8.5 & 13 & 5.3 \\
\hline Parotid $^{\star * *}$ & - & - & 12 & 9.3 & 12 & 4.9 \\
\hline Ear** & - & - & 8 & 6.2 & 8 & 3.2 \\
\hline
\end{tabular}

${ }^{*} p<0.05 ;{ }^{* *} p<0.01 ;{ }^{* *} p<0.001$ (Source: Individual medical records). NOTE: The percentages are obtained from the total of each septic process Odontogenic: 114, non-odontogenic: 129, total: 243.

tabla 5 relaciona ambos aspectos; se observa que la estancia osciló entre 1 y 45 días con un promedio de 7,6 días necesarios para un tratamiento antimicrobiano convencional. Se encontró que el mayor promedio obtenido con respecto a la estancia ocurrió en los pacientes que consumieron aminoglucósidos con ciprofloxacina, ya que alcanzaron una media de 22,5 días con una desviación estándar de 16,15 días; esto se explica por uno de los pacientes a quien se le suministró la combinación tuvo una estancia de 45 días, favorecido por la gravedad del proceso y la no conclusión diagnóstica, lo que propició que se aplicaran tres ciclos de tratamiento antimicrobiano y el último fue la combinación antes expresada.
Hospital strategy evidently leads the professional to be familiar with the effectiveness of the treatment given. Table 5 links both aspects. This shows that staging fluctuated between 1 and 45 days with an average of 7.6 days being necessary for conventional antimicrobial treatment. The greatest average obtained with regard to stay occurred in patients that consumed aminoglycosides with ciprofloxacin, as they reached an average of 22.5 days with a standard deviation of 16.15 days. This explains why one of the patients who was given a combination had a stay of 45 days, which was influenced by the seriousness of the process, and by not having a diagnostic conclusion. This led to three cycles of 
Tabla 4. Antimicrobianos utilizados y estancia de los pacientes en la institución hospitalaria

\begin{tabular}{|c|c|c|c|c|}
\hline \multirow[t]{2}{*}{ Antimicrobianos } & \multirow{2}{*}{$\begin{array}{l}\text { Pacientes } \\
\text { (número) }\end{array}$} & \multicolumn{3}{|c|}{ Estancia (en días) } \\
\hline & & Promedio & Mínimo & Máximo \\
\hline Penicilina y metronidazol & 66 & $6,89 \pm 3,54$ & 3 & 19 \\
\hline Penicilina* & 33 & $8,18 \pm 4,25$ & 3 & 20 \\
\hline Cefalosporina & 24 & $8,04 \pm 4,71$ & 3 & 21 \\
\hline Cefalosporina y metronidazol & 9 & $7,78 \pm 1,99$ & 5 & 12 \\
\hline Aminoglucósidos** & 19 & $8,26 \pm 5,81$ & 1 & 28 \\
\hline Aminoglucósidos con metronidazol** & 12 & $5,92 \pm 1,56$ & 3 & 9 \\
\hline Tetraciclina & 13 & $6,46 \pm 1,81$ & 4 & 10 \\
\hline Eritromicina & 14 & $5,22 \pm 2,05$ & 3 & 8 \\
\hline Ciprofloxacina & 4 & $8,50 \pm 1,91$ & 7 & 11 \\
\hline Cotrimoxazol** $^{* *}$ & 20 & $7,45 \pm 3,90$ & 1 & 18 \\
\hline Cloranfenicol & 19 & $5,49 \pm 2,40$ & 3 & 12 \\
\hline Vancomicina & 4 & $13,25 \pm 5,56$ & 5 & 17 \\
\hline Aminoglucósidos con ciprofloxacina & 4 & $22,25 \pm 16,15$ & 7 & 45 \\
\hline Oxacillin & 4 & $7,25 \pm 3,10$ & 3 & 10 \\
\hline Ketoconazol & 2 & $12,00 \pm 0,00$ & 12 & 12 \\
\hline Ninguno & 1 & $10,00 \pm 0,00$ & 10 & 10 \\
\hline Total & 243 & $7,6 \pm 4,64$ & 1 & 45 \\
\hline
\end{tabular}

Table 4. Antimicrobial agent used and hospital stay of patient

$\begin{array}{lc}\text { Antimicrobial agent } & \begin{array}{c}\text { Patient } \\ \text { (number) }\end{array} \\ \text { Penicillin and metronidazole } & 66 \\ \text { Penicillin* } & 33 \\ \text { Cephalosporin } & 24 \\ \text { Cephalosporin and metronidazole } & 9 \\ \text { Aminoglycoside** } & 19 \\ \text { Aminoglycoside with metronidazole** } & 12 \\ \text { Tetracycline } & 13 \\ \text { Erythromycine } & 14 \\ \text { Ciprofloxacin } & 4 \\ \text { Cotrimoxazole** } & 20 \\ \text { Chloramphenicol } & 19 \\ \text { Vancomycin } & 4 \\ \text { Aminoglycosides with ciprofloxacin } & 4 \\ \text { Oxacillin } & 4 \\ \text { Ketoconazole } & 2 \\ \text { None } & 1 \\ \text { Total } & 243 \\ & \end{array}$

\begin{tabular}{ccc} 
Stay (days) \\
\hline Average & Minimum & Maximum \\
$6.89 \pm 3.54$ & 3 & 19 \\
$8.18 \pm 4.25$ & 3 & 20 \\
$8.04 \pm 4.71$ & 3 & 21 \\
$7.78 \pm 1.99$ & 5 & 12 \\
$8.26 \pm 5.81$ & 1 & 28 \\
$5.92 \pm 1.56$ & 3 & 9 \\
$6.46 \pm 1.81$ & 4 & 10 \\
$5.22 \pm 2.05$ & 3 & 8 \\
$8.50 \pm 1.91$ & 7 & 11 \\
$7.45 \pm 3.90$ & 1 & 18 \\
$5.49 \pm 2.40$ & 3 & 12 \\
$13.25 \pm 5.56$ & 5 & 17 \\
$22.25 \pm 16.15$ & 7 & 45 \\
$7.25 \pm 3.10$ & 3 & 10 \\
$12.00 \pm 0.00$ & 12 & 12 \\
$10.00 \pm 0.00$ & 10 & 10 \\
$7.6 \pm 4.64$ & 1 & 45
\end{tabular}

Source: Individual medical records.

Le siguen en orden de frecuencia los que utilizaron vancomicina y ketoconazol, que estuvieron hospitalizados entre 13,25 y 12 días respectivamente.

La estancia de los pacientes tratados con el resto de los antimicrobianos osciló entre los 5,22 y 8,50 días.

Conviene destacar que no fue posible confeccionar un protocolo de tratamiento al realizar este estudio (para comparar resultados), pues la disponibilidad de los medicamentos resultó ser muy variable y fue necesario ajustarse a ellos; sin embargo, la penicilina y el metronidazol mantuvieron relativa estabilidad de modo que, a los pacientes que padecían procesos odontógenos se les trató con antimicrobial treatment being given, the last one being the combination previously mentioned.

This is followed in order of frequency by those that used vancomycin and ketoconazole who were hospitalized between 13.25 and 12 days respectively. The stay of the patients treated with the remaining antimicrobial drugs varied between 5.22 and 8.50 days respectively.

It should be pointed out that drawing up a treatment protocol was not possible on carrying out this study (in order to compare results), as the availability of drugs was very variable and adjustments had to be made because of this. How- 
Tabla 5. Complicaciones presentadas por los pacientes según su etiología del proceso séptico

\begin{tabular}{|c|c|c|c|c|c|c|}
\hline \multirow[b]{2}{*}{ Complicaciones } & \multicolumn{2}{|c|}{ Odontógenos } & \multicolumn{2}{|c|}{ No odontógenos } & \multicolumn{2}{|c|}{ Total } \\
\hline & $\mathbf{N}^{\circ}$ & $\%$ & $\mathbf{N}^{\circ}$ & $\%$ & $\mathbf{N}^{\mathbf{0}}$ & $\%$ \\
\hline Angina de Ludwing & 1 & 0,9 & - & - & 1 & 0,4 \\
\hline Absceso parafaringeo & 1 & 0,9 & - & - & 1 & 0,4 \\
\hline Absceso pulmonar & - & - & 2 & 1,6 & 2 & 0,8 \\
\hline Fallecido (multiorgánico) & - & - & 1 & 0,8 & 1 & 0,4 \\
\hline Ninguna & 110 & 96,5 & 123 & 95,3 & 233 & 95,9 \\
\hline Total & 114 & 46,9 & 129 & 53,1 & 243 & 100,0 \\
\hline
\end{tabular}

Table 5. Complications presented by the patients according to etiology of the septic process

Complication
Ludwig's angina
Osteomielitis
Parapharyngeal abscess
Lung abscess
Death (multiorgan)
Pneumonia due to staphylococcus
Death due to another cause
None
Total

Source: Individual medical records

\begin{tabular}{cc}
\multicolumn{2}{c}{ Odontogenic } \\
$\mathbf{N}^{\circ}$ & $\%$ \\
1 & 0.9 \\
2 & 1.8 \\
1 & 0.9 \\
- & - \\
- & - \\
- & - \\
- & - \\
110 & 96.5 \\
114 & 46.9
\end{tabular}

Total

$\begin{array}{cc}\mathbf{N}^{\circ} & \% \\ 1 & 0.4 \\ 2 & 0.8 \\ 1 & 0.4 \\ 2 & 0.8 \\ 1 & 0.4 \\ 1 & 0.4 \\ 2 & 0.8 \\ 233 & 95.9 \\ 243 & 100.0\end{array}$

esta combinación excepto a los que refirieron alergia a la penicilina, en cuyo caso fue sustituida por cefazolina, un aminoglucósido u otro antimicrobiano.

La selección del antimicrobiano para tratar la sepsis no odontógena se subordinó a su etiología y disponibilidad. Fue reservado el uso de fármacos antiestafilocóccicos para la celulitis facial por forúnculos y sepsis posquirúrgica. Se dispuso preferentemente de cloranfenicol y sulfaprim. En caso de alergia o de mayor gravedad se seleccionaron cefalosporinas o aminoglucósidos. La vancomicina se mantuvo guardada para últimos recursos. El ketoconazol se administró para tratar la candidiasis orofaríngea en dos pacientes $(0,8 \%)$.

La terapia antimicrobiana fue complementada con drenaje quirúrgico cuando fue necesario; también se complementó con la extracción dentaria o acceso cameral en los procesos de origen dentario.

\section{Discusión}

La alta frecuencia con que se presentan los procesos sépticos, a pesar de las posibilidades de diagnóstico, control y tratamiento en la atención primaria, obliga a considerarla un problema de salud.

Un estudio con resultados similares fue realizado en el Hospital "General Calixto García", de nuestra Capital por Díaz Veliz en el ever, penicillin and metronidazole were relatively stable, to the extent that patients with odontogenic processes were treated with this combination, with the exception of those that reported penicillin allergies, in which case this was substituted by cefazolin, an aminoglycoside or another antimicrobial agent.

The choice of antimicrobial agent for treating non-odontogenic sepsis depended on etiology and availability. Antistaphylococcus drugs were reserved for facial cellulitis because of boils, and postsurgical sepsis. Chloramphenicol and sulfaprim were chiefly available. In cases of allergy, or cases that were more serious, cephalosporins or aminoglycosides were chosen. Vancomycin was kept as a last resort. Ketoconazole was administered for treating oropharyngeal candidiasis in two patients (0.8\%).

Antimicrobial therapy was complemented with surgical drainage when necessary. It was also complemented with dental extraction or chamber access in processes with a dental origin.

\section{Discussion}

The high frequency with which these septic processes arise, despite the possibilities regarding diagnosis, control 
que se obtuvo que el $21,3 \%$ de los 286 pacientes hospitalizados padecían de infección en el área cervicofacial. Escudes, ${ }^{3}$ alega que representan entre $20-25 \%$ de los pacientes que ingresan al Servicio de Cirugía Maxilofacial, cifra que se corresponde con la obtenida en nuestra investigación.

Históricamente la etiología odontógena ha lidereado la enfermedad séptica de cabeza y cuello, no ocurre así en nuestro estudio. Para Kimura, ${ }^{7}$ representa el $88,9 \%$ de los pacientes.

Existen algunas razones importantes que ayudan a explicar esta discrepancia:

- Los procesos sépticos no odontógenos incluyen una variedad de afecciones; cada una con comportamiento epidemiológico diferente $y$, una de ellas, la celulitis facial estafilicócica (secundaria a lesión de piel), ha experimentado una alta incidencia en los últimos años, he incluso ha sido la causa de la mayoría de los ingresos de origen no odontógeno en nuestro servicio. La alta frecuencia de la sepsis estafilocóccica queda demostrada en el Informe anual de laboratorio de Microbiología al Comité de infecciones para los años en estudio.

- Por otra parte, los pacientes ingresados presentan los signos y síntomas que hacen que se afirme que tienen los procesos sépticos más graves o complicados y precisan de atención hospitalaria; por tanto, no se excluye la existencia de gran número de pacientes que cursan la enfermedad de forma ambulatoria y que no se incluyen en nuestro estudio.

- El personal médico del primer nivel de atención (estomatólogos y médicos de familia) y la población en general están más familiarizados con el tratamiento de los procesos odontógenos, por lo que no acuden con más frecuencia a nuestro servicio porque consideran que pueden solucionarlo a su nivel.

Adviértase que existen criterios, ${ }^{8}$ que niegan la influencia del sexo en la susceptibilidad a la infección; sin embargo, otros autores,, 10 con inclusión nuestra, abogamos por una ligera supremacía del sexo masculino, por ser este un grupo con características particulares que condicionan un mayor riesgo al estar más expuestos a traumas por accidentes y riñas, mayor relación con trabajos sucios, en exteriores y con medios contaminados, $y$, de forma general por hábitos de higiene mas descuidados. La barba y la frecuencia del afeitado ayudan a explicar la elevada cifra de los varones en los procesos no odontógeno, al aumentar considerablemente el riesgo de forúnculos y foliculitis.

Los resultados obtenidos respecto a edad y sexo predominantes coinciden con los obtenidos por Díaz Veliz y Rodríguez Calzadilla. ${ }^{11}$ Es precisamente este grupo de edad el más activo biológica y socialmente, y por tanto más susceptible al padecimiento en estudio.

Se observa una tendencia a la disminución de los procesos sépticos según aumenta la edad con una mayor acentuación en los odontógenos, fácilmente explicable por el natural deterioro del aparato dentario y el incremento de las enfermedades con parotiditis y sobreinfecciones de neoplasias. ${ }^{12}$

Diversos autores, ${ }^{11-13}$ también encontraron la región submandibular como la más afectada en la sepsis de origen odontógeno.

Las referencias del empleo de la combinación de penicilina y metronidazol no son escasas ya que varios investigadores consultados, ${ }^{14-17}$ destacan sus ventajas y eficacia. and treatment in primary care, means that considering this a health problem is necessary.

A study with similar results was carried out in the "Hospital General Calixto García" in our capital by Díaz Veliz, which showed that $21.3 \%$ of the 286 patients who were hospitalized suffered from infection in the cervicofacial area. Escudes 3 claims that they make up 20-25\% of patients who are admitted to Maxillofacial Surgery departments, a figure that agrees with the one obtained in our investigation.

Historically, it is processes with an odontogenic origin that have headed septic disease of the head and neck, but this did not occur in our study. For Kimura ${ }^{7}$ this represents $88.9 \%$ of the patients.

There are some important reasons that help to explain this discrepancy:

- Non-odontogenic septic processes include a variety of conditions, each with different epidemiological behavior and one, staphylococci facial cellulitis (derived from skin lesions), has experienced a high incidence over the last years, and it has even been the cause of most of the admittances to our department with a non-odontogenic origin. The high rate of staphylococcus sepsis has been demonstrated in the annual reports sent by the Microbiology Laboratory to the Infections Committee for the years that have been studied.

- Moreover, the patients admitted have signs and symptoms that suggest they have the more serious or complicated septic processes, and that they require hospital care. And that, therefore, the existence of large numbers of patients that experience the disease on an outpatient basis and that are not included in our study cannot be excluded.

- Medical personnel at a primary care level (dentists and family doctors) and the population in general are more familiar with the treatment for odontogenic processes and, as a result, they do not attend our department regularly because they believe that they can solve the process themselves.

It should be mentioned that there are criteria8 that deny any gender influence in infection susceptibility. However, other authors, 10 including ourselves, defend that there is a slight male supremacy, as this is a group with special characteristics and with greater risks, because they are more exposed to trauma as a result of accidents and disputes, they have a greater association with dirty jobs either outside or with contaminated medium, and in general because of slacker hygiene. Having a beard and shaving frequency helps to explain the high number of males with non-odontogenic processes, as the risk of boils and folliculitis increases.

The results obtained with regard to age and sex predominance coincide with those obtained by Díaz Veliz and Rodríguez Calzadilla." It is precisely this age group which is more active biologically and socially, and therefore more susceptible to this ailment in the study.

A tendency for septic processes to reduce as age increases can be observed together with a greater accentuation on 
La penicilina constituye aún la primera elección en el tratamiento de las infecciones odontogénicas. A pesar de los inconvenientes de su administración (vía y frecuencia) la penicilina $G$ conserva un valor inestimable en las infecciones graves al ser su espectro de acción reducido y coincidir satisfactoriamente en los encontrados de modo habitual en las infecciones odontogénicas. ${ }^{16} \mathrm{El}$ metronidazol se ha utilizado cada vez más en todo el mundo debido a que la resistencia es poco común y a causa de sus propiedades farmacológicas que le permiten penetrar abscesos e inhibir bacterias en un ambiente anaerobio. ${ }^{18}$

Los procesos no odontógenos precisaron del reconocimiento etiológico para emprender la terapia farmacológica, y la evolución natural de la enfermedad indicó la necesidad del drenaje quirúrgico o no.

Sin embargo, en los procesos odontógenos, realizar la exodoncia del diente causal es imperativo si no existen posibilidades futuras de tratamiento conservador. Solo la limitación a la apertura bucal y la no instalación de un tratamiento antimicrobiano pueden retardar o posponer la exodoncia, aunque se reconoce que cambiar la terapia antimicrobiana apropiada con la supresión de la causa dental y/o drenaje quirúrgico temprano favorece y acelera la resolución del proceso (Kimura, ${ }^{7}$ Kpemissi, ${ }^{15}$ Scheffer, ${ }^{19}$ Miconi20), éste no es un proceder común en el primer nivel de atención. De forma casi general muchos estomatólogos recomiendan aplazar toda investigación quirúrgica incluyendo la extracción dentaria hasta que haya desaparecido el período agudo de la celulitis por considerarlo peligroso bien por desconocimiento o temor y abusan del empleo de la terapia antimicrobiana, lo que provoca la no resolución y si la agudización del proceso que puede llegar al extremo de considerar la hospitalización del paciente tratado ambulatoriamente de forma incorrecta. ${ }^{11-21}$

Kimura, ${ }^{7}$ en su serie de 147 pacientes refiere que el $75 \%$ de ellos se cursó con antibióticoterapia parenteral y el resto requirió drenaje u otro proceder quirúrgico y solo expresa que un paciente se complicó con una trombosis séptica del seno cavernoso.

\section{Conclusiones}

Es imprescindible establecer un diagnóstico y tratamiento precoces de las infecciones cervicofaciales con vistas a disminuir la hospitalización que ellas pueden derivar. Aunque estos procesos sépticos constituyen una afección potencialmente grave, la mayoría de quienes la padecen se recuperan con adecuado tratamiento.

\section{Bibliografía}

1. Mandell GL. Introducción al VIH y trastornos asociados. En: Bennett JC, Plum F. Cecil. Tratado de Medicina Interna. 20 ed. México: Mc Graw-Hill Interamericana 1998;2118.

2. Mandell GL. Introducción a las enfermedades antimicrobianas. En: Bennett JC, Plum F. Cecil Tratado de Medicina Interna. 20 ed. México: Mc Graw-Hill Interamericana 1998;1678-9. odontogenic processes, easily explained by the natural deterioration of the dental apparatus and the increase in diseases such as parotiditis and extra infections from neoplasms. ${ }^{12}$

Many authors ${ }^{11-13}$ also found the submandibular region to be the most affected by sepsis of odontogenic origin.

The references to using a combination of penicillin and metronidazole are far from few, as many of the investigators referred to $0^{14-17}$ stress the advantages and efficiency of this combination.

Penicillin is the first choice for treating odontogenic infections. In spite of the inconveniences of its administration (method and frequency) penicillin $G$ is still of inestimable value for serious infections, as its action spectrum is reduced, and it coincides satisfactorily in those habitually found in odontogenic infecctions. ${ }^{16}$ Metronidazole has been used increasingly over the world due to resistance being unusual, and because of its pharmacological properties that permit penetrating abscesses and repressing bacteria in an anaerobic environment. ${ }^{18}$

The non-odontogenic processes did not require the identification of the etiological condition for pharmacological therapy to be started, and the natural evolution of the disease indicated the need for surgical drainage or not.

However, in odontogenic processes, removing the tooth causing the infection is imperative if there is no possibility for conservative treatment in the future. The extraction should only be delayed or postponed because of limited oral aperture or because antimicrobial treatment has not been started, although it is known that changing appropriate antimicrobial therapy for suppression of the tooth causing the infection and/or prompt surgical drainage, favor and accelerate the resolution of the process (Kimura, ${ }^{7}$ Kpemissi, ${ }^{15}$ Scheffer, ${ }^{19}$ Miconi). ${ }^{20}$ This is not a common procedure at primary care level. Dentists will nearly always recommend delaying all surgical investigation, including dental extraction, until the acute cellulitis period has disappeared, as it is considered dangerous, either because of a lack of familiarity or fear, and antimicrobial therapy is misused. This leads to the nonresolution and the deterioration of the process, which can end with the hospitalization of patients treated incorrectly as outpatients. ${ }^{11-21}$

Kimura, ${ }^{7}$ reported that $75 \%$ of the 147 patients is his series recovered with parenteral antibiotic therapy and the remainder required drainage or another surgical procedure, and only one patient is mentioned with septic thrombosis complications of the cavernous sinus.

\section{Conclusion}

Early diagnosis and treatment is essential for infections of the head and neck with regard to reducing the hospitalization of patients that this can lead to. Although these septic processes represent a potentially serious condition, most of these patients will recover with adequate treatment. 
3. Escudes O. Infecciones en el área cervicofacial. Infecciones odontogénicas. En: Pericot J Actualidades clínico-terapéuticas en Cirugía Máxilo Facial. Barcelona: J. Uniach \& CIA 1997;221-45.

4. Archer GL. Infecciones estafilocóccicas. En: Bennett JC, Plum F, Cecil. Tratado de Medicina Interna. 20ed. México: Mc Graw-Hill Interamericana 1998;1854 -9.

5. Bryant RE. Skia and subcutaneous infections. En: Stein JH. Internal Medicin. $4^{\text {th }}$ ed. St Louis: Mosby; 1994;1907-15.

6. Berini Aytes L, Garatea Grelgo J, Gay Escoda C. La infección odontogénica: concepto, etiopatogenia, bacteriología y clínica. En Gay Escoda C, Berini Aytes L. Cirugía bucal. 1ra ed. Madrid: Ergon 1999;125 -49 .

7. Kimura AC, Pien FD. Head and neck cellulitis in hospitalized adults. Am J Otolaryngol 1993;14:343-49.

8. Rush KL, Haller LT. Patient factors and central line infection. Clin Nurs Res 1995; 4:397-410.

9. Richar C. The treatment of dog bite injuries, especially those the face. Plast Reconstr Surg 1989;64:5-11.

10. Braham RL. Management of dental trauma in children and adolescents. J Trauma 1987;27:10-6.

11. Rodríguez Calzadilla OL. Celulitis facial odontógena. Rev Cubana Estomatol 1997; 34:15-20.

12. Márquez Mateo M, García Fernández R, Puche Torres M, Masón Sánchez P, Vidal Torres A, Pérez Flores D y cols. Incidencias y etiopato- genia del cáncer de cavidad oral en la región de Murcia. Rev Esp Cir oral Maxilofacial 1995; 17:22-27.

13. Díaz Fernández JM, Gutiérrez Macias I. Angina de Ludwing. Análisis de 11 casos. Rev Cubana Estomatol 1996;33:101-4.

14. Davis Allan J, Jr. Combinaciones de antibióticos. Actualización sobre antibióticos. Clin Med North Am 1987;1145-58.

15. Kpemissi E. Cervicofacial cellulitis of oral and dental origin: study of 26 cases at the Lone University Hospital. Rev Laryngol Otol Rhinol (Bond) 1999;116:195-7.

16. Marcello EJ. Fundamentos farmacológicos de la terapéutica antimicrobiana. Av. Odontoestomato 1997;13(Suppl A):53-75.

17. Nielsen TR, Clement F, Andrea Sen. UR. Mediastinitis, a rare complications of a peritonsilar abscess. J Laryngol and Otology 1996;110:175-76.

18. Neu Harold C. Conceptos generales sobre quimioterapia de enfermedades infecciosas. Clin Med Nort Am 1987;68:1115-29.

19. Scheffer P, Ouazzani A, Esteban J, Lerondeau JC. Serious cervicofacial infections of dental origin. Rev Stomatol Chir Maxillofac 1989;90:115-8.

20. Miconi M, Gallesio C, Berrone S. Clinico-Therapeutic observations on a series of case of odontogenic abscesses and phlegnons. Minerva Stomatol 1991;40: 641-9.

21. Santos Pena MA, Betancourt García A, Queiroz Enrique M, Curberia Hernández EM, Santana Fernández D. Manual de terapéutica antimicrobiana en Estomatología. Temas de actualización. Rev Cubana Estomatol 1999;36:103-50. 